\title{
EFECTO DE LAS CONDICIONES DE SECADO SOBRE LA CINÉTICA DE DESHIDRATACIÓN DE LAS HOJAS DE MORERA (Morus alba $)^{1}$
}

\author{
María Lourdes Pineda-Castro ${ }^{2}$, Alejandro Chacón-Villalobos ${ }^{3}$,Gendi Cordero-Gamboa ${ }^{4}$
}

\begin{abstract}
RESUMEN
Efecto de las condiciones de secado sobre la cinética de deshidratación de las hojas de morera (Morus alba). El objetivo del presente trabajo fue determinar las cinéticas de deshidratación de la morera bajo diferentes condiciones de temperatura, velocidad y humedad del aire, entre junio y diciembre del 2006, en Costa Rica. Forraje de morera (Morus alba) de 75 días fue empleado como sustrato. Se evaluó la cinética de deshidratación en un secador convectivo de resistencias eléctricas de bandeja única con diámetro de 0,22 m y carga de $122 \pm 1 \mathrm{~g}$ hasta una humedad del $5 \%$. La evaluación se realizó para tres temperaturas de secado $\left(60{ }^{\circ} \mathrm{C}, 75^{\circ} \mathrm{C}\right.$ y 90 $\left.{ }^{\circ} \mathrm{C}\right)$, dos velocidades de aire $(1,0$ y $2,5 \mathrm{~m} / \mathrm{s})$ y dos humedades del aire (12 g/kg aire seco y $45 \mathrm{~g} / \mathrm{kg}$ aire seco), para un total de 12 tratamientos triplicados. Las curvas de secado correspondientes se estudiaron según el modelo de decaimiento exponencial de Newton. Se obtuvieron gráficos de velocidad de secado en función del tiempo y la humedad. Las cinéticas de deshidratación presentaron la curva típica del modelo de Newton, con altos coeficientes de correlación. Existió un efecto triple significativo $(\mathrm{p}<0,05)$ entre la temperatura, la velocidad y la humedad del aire sobre el tiempo de secado del sustrato; la variable más influyente fue la temperatura, seguida de la velocidad y por último la humedad del aire. El aumento de cualquiera de estas variables implicó una disminución del tiempo de secado. El efecto de la temperatura sobre éste fue más pronunciado a una velocidad del aire de $1 \mathrm{~m} / \mathrm{s}$.
\end{abstract}

Palabras clave: Forraje, alimentación animal, procesamiento, modelado, pienso.

\begin{abstract}
Effect of drying conditions upon dehydration kinetic of mulberry (Morus alba). The objective of this work was to determine the dehydration kinetics of mulberry under different conditions of temperature, speed and air humidity, between June and December 2006 in Costa Rica. White mulberry leaves (Morus alba) with an age of 75 days were used as substrate for the drying process. The drying kinetics was investigated in a electric convective dryer with a single tray (diameter of $0.22 \mathrm{~m}$ ) loaded with $122 \pm 1 \mathrm{~g}$ of mulberry leaves per batch, maintaining the process until a humidity content of $5 \%$ was reached. Three drying air temperatures $\left(60{ }^{\circ} \mathrm{C}, 75^{\circ} \mathrm{C}\right.$ and $\left.90{ }^{\circ} \mathrm{C}\right)$, two fixed airflow rates $(1 \mathrm{~m} / \mathrm{s}$ and $2.5 \mathrm{~m} / \mathrm{s})$ and two air humidity contents (12 $\mathrm{g} / \mathrm{kg}$ dry air and $45 \mathrm{~g} / \mathrm{kg}$ dry air) were evaluated for a total of 12 treatments. The drying data were fitted to the Newton mathematical model. Graphics for drying velocity vs. time and vs. humidity were also obtained. The drying kinetics was in good agreement with a typical Newton model showing high correlation coefficients. There was a significant effect $(p<0.05)$ of temperature, velocity and humidity of air upon drying, where temperature was the most influential of them all, followed by velocity and finally by air humidity. Increasing any of these parameters implied a reduction of the drying time. The effect of temperature on drying was most pronounced at a air speed of $1 \mathrm{~m} / \mathrm{s}$.
\end{abstract}

Key words: Forage, animal feeding, processing, modelling, feedstock.

\footnotetext{
1 Recibido: 17 de setiembre, 2008. Aceptado: 16 de noviembre, 2009. Inscrito en Vicerretoría de Investigación N N $^{\circ}$ 737-A5-074 y N $735-A 2-$ 502, Universidad de Costa Rica.

2 Escuela de Tecnología de Alimentos. Facultad de Ciencias Agroalimentarias. Universidad de Costa Rica. San José, Costa Rica. maria. pinedacastro@ucr.ac.cr

3 Estación Experimental Alfredo Volio Mata. Facultad de Ciencias Agroalimentarias. Universidad de Costa Rica. Cartago, Costa Rica. alejandro.chacon@ucr.ac.cr

4 Laboratorios Griffith Centro América, S.A. gcordero@griffithlaboratories.com
} 


\section{INTRODUCCIÓN}

La ganadería enfrenta en épocas recientes la necesidad de adoptar políticas de sostenibilidad y eficiencia con el objetivo de mantener su competitividad (Boschini, 2000). La alimentación de los animales durante el proceso agrícola no es ajena a esta realidad, razón por la que son diversas las fuentes nutricionales estudiadas desde antaño, tales como forrajes, soja, maíz y hasta concentrados de proteína unicelular (Chacón 2004).

En el área mesoamericana es especialmente importante la evaluación y búsqueda de nuevos cultivos forrajeros, categoría en la que tienen auge reciente los forrajes arbóreos de alta producción de biomasa y sus valiosos contenidos de nutrientes (Estévez et al. 2004, Boschini 2006). Los arbustos leñosos son una opción forrajera más eficiente en la generación de biomasa que las gramíneas, destacándose la morera (Morus alba) por su gran adaptabilidad a la zona tropical centroamericana (Rodríguez et al. 1994) y fácil integración en los sistemas pecuarios (Boschini 2003, García y Ojeda 2004). Se le cultiva con diversos fines, tales como alimento para el gusano de la seda (Bombyx mori), el consumo humano de sus frutos o infusiones, como forraje, y hasta con fines decorativos (Duke 1983, Sánchez 1999). La variedad blanca (M. alba) es la que se cultiva en Costa Rica, dónde, a pesar de abarcar un área reducida, muestra rendimientos superiores a las $30 \mathrm{t} / \mathrm{ha}$ /año de material verde (Boschini 2003). Se le destina allí exitosamente a la alimentación de bovinos y caprinos (Boschini 2008) , $^{5}$ estableciéndose rendimientos de producción láctea de $2,3 \mathrm{~kg} / \mathrm{animal} /$ día en cabras con dietas fundamentadas en una tercera parte de morera, y de $12,3 \mathrm{~kg} / \mathrm{animal} /$ día con $65 \%$ de morera fresca en la dieta en ganado vacuno (Boschini 2003).

La morera se caracteriza por ser un forraje de excelente palatabilidad (Ortiz 1992, Boschini 2006) y un alto consumo (Benavidez et al. 1994, Boschini et al. 1998). Se caracteriza además por una digestibilidad in vitro de la materia seca entre $70 \%$ y $80 \%$ (Ortiz 1992), un contenido de proteína cruda entre 14 y 22 $\%$ en base seca (Piccioni 1970) y una producción de

\footnotetext{
5 Boschini, C. 2008. Importancia del cultivo y secado de la Morera (Morus alba) en Costa Rica. Universidad de Costa Rica, Estación Experimental de Ganado Lechero Alfredo Volio Mata. Cartago. Comunicación personal.
}

biomasa que se estima superior a las 15 toneladas de materia seca por hectárea al año (Nieves et al. 2006). Boschini (2000) reporta además un alto contenido de humedad que ronda un $75 \%$, así como valores de fibra cruda de $19 \%$ estimados en hoja, $1,48 \mathrm{Mcal} / \mathrm{kg}$ de energía neta, 32,83\% de fibra neutro detergente, $8,66 \%$ de hemicelulosa, $24,17 \%$ de fibra ácido detergente, $18,76 \%$ de celulosa, $5,44 \%$ de lignina y 16,78 $\%$ de cenizas en materia seca. La calidad nutricional expuesta, sumada a la respuesta productiva de los rumiantes y a su carácter cosmopolita, son causales directos de un acentuado uso de este forraje en sistemas productivos de la región mesoamericana (Alfaro et al. 1997).

El secado de forrajes de alto contenido de proteína y alto valor energético como la morera tiene una importante repercusión técnica y económica para el productor (Boschini 2008). Al eliminar la humedad, se logran concentrados de alta calidad nutricional útiles en la elaboración de alimentos balanceados o en suplementos proteicos, los cuales probablemente presenten un menor costo económico que otras fuentes tradicionales de suplementación como la soya, lo cual implicaría un impacto directo en la sostenibilidad de los sistemas agrosilvopastoriles (Elizondo 2004).

El secado es una de las operaciones unitarias más comunes en la preservación de fuentes nutricionales (Geankoplis 1998), lo que implica simultáneamente una transferencia de calor y masa acompañada por un cambio de fase (Barbanti et al. 1994). A nivel experimental, el secado de las hojas de morera se realiza con aire caliente que aporta calor al producto por convección (Sharma et al. 2003, Brennan et al. 1998), utilizando temperaturas que oscilan entre 60 y $90{ }^{\circ} \mathrm{C}$ (Bamikole et al. 2005, Jirakitkul 2007, Murakami 2003). Durante este proceso se disminuye la actividad de agua y, con ella, la degradación bioquímica y microbiológica durante el almacenamiento (Doymaz 2004). La velocidad de deshidratación depende de factores determinantes en la cinética de secado como la fisicoquímica y la forma del producto (Krokida et al. 2003), así como de la temperatura, velocidad y humedad del aire de secado (Sharma et al. 2003).

La eliminación del agua ocurre generalmente en una serie de etapas diferenciadas por la velocidad

\footnotetext{
6 Elizondo, J. 2004. Aspectos técnicos nutricionales de la Morera (Morus alba). Estación Experimental Alfredo Volio Mata. Comunicación personal.
} 
de secado (Singh y Heldman 1993). Después de un corto período de estabilización inicial donde el sólido está a baja temperatura y la velocidad de evaporación aumenta a medida que sube la temperatura, se registra una etapa de velocidad de secado constante, donde la temperatura de la superficie alcanza su valor de equilibrio (temperatura de bulbo húmedo del aire), lo que provoca una reducción importante del contenido de agua a temperatura y velocidad de secado constante, mientras la superficie del sólido se mantiene saturada de agua que migra desde el interior a la misma velocidad que ocurre la evaporación en la superficie. Al alcanzarse la humedad crítica inicia el primer período de velocidad decreciente, generalmente lineal, comenzando a secarse la superficie. La temperatura de la superficie empieza a elevarse sostenidamente hasta que se aproxima a la de bulbo seco del aire cuando el producto se acerca al estado de sequedad. Acontece entonces el segundo período de velocidad decreciente; se desplaza el plano de evaporación hacia el interior y disminuye con celeridad la velocidad de secado hasta alcanzar la humedad de equilibrio ${ }^{7}$ del producto. En algunos materiales, el primer período de velocidad decreciente puede no existir o bien constituir la totalidad del periodo de velocidad decreciente (Singh y Heldman 1993, Geankoplis 1998).

El estudio y la simulación de las cinéticas de secado por medio de ecuaciones matemáticas empíricas en función de los parámetros del proceso, es necesario para efectos de control y optimización de los procesos de secado (Vega et al. 2007). La literatura relacionada con la parametrización del secado de la morera, basándose en la optimización de tiempos y temperaturas ideales es escasa. El objetivo del presente trabajo fue determinar las cinéticas de deshidratación de la morera bajo diferentes condiciones de temperatura, de velocidad y humedad del aire, empleando un secador convectivo.

\section{MATERIALES Y MÉTODOS}

Este estudio se efectuó en el segundo semestre del año 2006 en las instalaciones de la Estación Experimental Alfredo Volio Mata de la Universidad de Costa

\footnotetext{
Contenido de humedad definido que alcanza un sólido bajo condiciones especificadas de temperatura y humedad del aire (Geankoplis 1998).
}

Rica (EEAVM), ubicadas en Ochomogo de Cartago, así como en las Instalaciones de la Planta Piloto del Centro Nacional de Ciencia y Tecnología de Alimentos (CITA) de la Universidad de Costa Rica, ubicadas en San Pedro de Montes de Oca, San José.

El forraje de morera se obtuvo por corta manual en los campos de cultivo ubicados en la EEAVM, que fueron especialmente plantados y segregados para tal efecto, contando los brotes con una edad de corta de 75 \pm cinco días. Inmediatamente después del corte, que se efectuó en las primeras horas de la mañana, el forraje fue remitido, empacado en sacos opacos y al resguardo de la luz solar, al CITA, ubicado a $15 \mathrm{~km}$ de distancia del lugar de cultivo, en un viaje que no sobrepasó los 15 minutos de duración en ninguno de los casos.

Al arribar los cortes a la planta piloto, éstos se lavaron con agua para eliminar impurezas y residuos, procediéndose luego a su escurrido completo y a la separación manual de las hojas, constituyendo estas últimas el sustrato a ser deshidratado. Las hojas seleccionadas fueron subdivididas en fragmentos de aproximadamente $3 \times 4 \mathrm{~cm}$. La humedad inicial de las muestras se determinó en todos los casos por medio de la metodología gravimétrica de estufa de vacío recomendada por Vega et al. (2007).

Los diferentes procesos de secado se efectuaron con un secador convectivo de resistencias eléctricas con bandeja única de diámetro equivalente a $0,22 \mathrm{~m}$ y equipado con monitores para la velocidad y temperatura del aire de secado (Figura 1). El secador contó con ámbitos operativos desde temperatura ambiente más $5^{\circ} \mathrm{C}$ hasta $160^{\circ} \mathrm{C}$, para una velocidad de aire desde

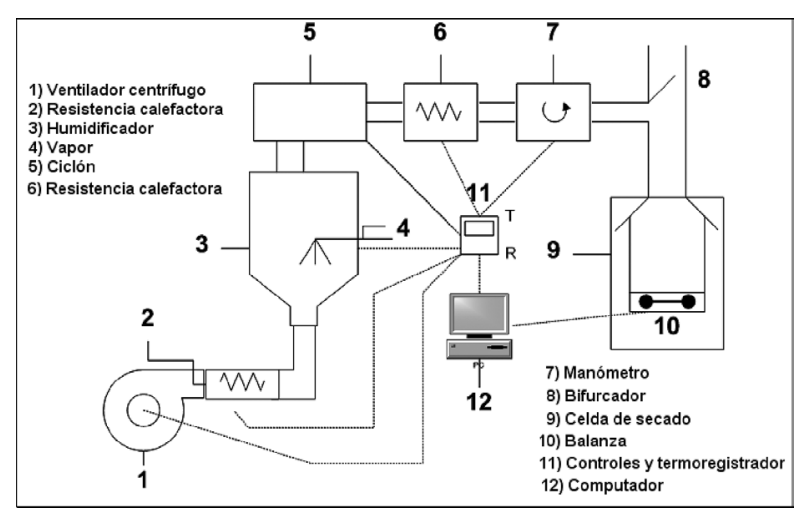

Figura 1. Diagrama del secador piloto del Centro de Investigaciones en Tecnología de Alimentos (CITA). San José, Costa Rica, 2008. 
$1,0 \mathrm{~m} / \mathrm{s}$ hasta $2,5 \mathrm{~m} / \mathrm{s}$, perpendicular a la bandeja, y a partir de una humedad ambiente hasta $150 \mathrm{~g}$ agua $/ \mathrm{kg}$ aire seco.

Se evaluaron los tiempos de secado de las hojas de morera hasta una humedad del 5\% con la combinación de tres temperaturas de secado $\left(60^{\circ} \mathrm{C}, 75^{\circ} \mathrm{C}\right.$ y $\left.90^{\circ} \mathrm{C}\right)$, dos velocidades del aire $(1,0$ y $2,5 \mathrm{~m} / \mathrm{s})$ y dos humedades del aire (12 g/kg aire seco, o humedad ambiente, y $45 \mathrm{~g} / \mathrm{kg}$ aire seco), para un total de 12 tratamientos. Cada uno de los tratamientos de secado se realizó por triplicado, se utilizó un lote diferente de forraje para cada réplica. La bandeja del secador recibió $122 \pm 1 \mathrm{~g}$ de muestra en cada una de las réplicas de los procesos experimentales de secado, para una carga de bandeja de $2,52 \mathrm{~kg} / \mathrm{m}^{2}$. La humedad inicial promedio de las muestras fue del $81 \%$.

Las muestras se colocaron según tratamientos en una cámara de secado conectada a un sistema neumático que permite conocer la masa del producto a través del tiempo, y se registraron los datos automatizadamente cada minuto.

Las cinéticas de secado se construyeron al graficar la relación de humedad en base seca en cualquier momento (X) dividida por la humedad en base seca inicial $\left(\mathrm{X}_{\mathrm{O}}\right)$, lo que se conoce como razón de humedad $\left(\mathrm{X} / \mathrm{X}_{\mathrm{O}}\right)$, en función del tiempo.

El cálculo de $\mathrm{X}$ y $\mathrm{X}_{\mathrm{O}}$ se realiza a través de las siguientes ecuaciones:

$X=\frac{P_{X}-\left[P_{X} *\left(100-h_{X}\right)\right] 100}{P_{X} *\left(100-h_{X}\right) / 100}=\frac{1}{1-\left(h_{X} / 100\right)}-1$

donde

$\mathrm{P}_{\mathrm{X}}$ es la masa en cualquier momento $(\mathrm{kg})$

$\mathrm{h}_{\mathrm{X}}$ es la humedad en base húmeda en cualquier momento (\%)

Las curvas de secado de las tres repeticiones de cada tratamiento se evaluaron con base en el modelo de Newton que expresa un decaimiento exponencial de la humedad con el tiempo de secado:

$$
\mathrm{X} / \mathrm{X}_{\mathrm{O}}=\mathrm{a}^{*} 10^{(-\mathrm{kt})}
$$

La ecuación $\mathrm{X} / \mathrm{X}_{\mathrm{O}}=\mathrm{a}^{*} 10^{(-\mathrm{kt})}$ se transformó linealmente al aplicar logaritmo a ambos lados:

$$
\log \left(\mathrm{X} / \mathrm{X}_{\mathrm{O}}\right)=-\mathrm{kt}+\log \mathrm{a}
$$

donde,

$\mathrm{t}$ : tiempo (min)

$\mathrm{k}$ : constante de velocidad $\left(\mathrm{min}^{-1}\right)$

$\log$ a: intersección con el eje de las $\mathrm{Y}$

El efecto de la temperatura, la humedad y la velocidad del aire de secado sobre el tiempo de deshidratación hasta $5 \%$ de humedad se analizó por medio de un análisis de varianza, con base en el programa JMP-SAS V.4. Adicionalmente, se construyeron los gráficos de velocidad de secado en función del tiempo y velocidad de secado en función de X, con 20 mediciones espaciadas regularmente a lo largo de todo el periodo experimental (Belghit et al. 2000), las que fueron suficientes para evidenciar las tendencias. La velocidad de secado en $\mathrm{kg} / \mathrm{h} \cdot \mathrm{m}^{2}(\mathrm{v})$ se calculó empleando la función:

$$
\begin{aligned}
& \mathrm{v}=\left(\mathrm{P}_{\mathrm{i}-1}-\mathrm{P}_{\mathrm{i}}\right) /\left[\left(\mathrm{t}_{\mathrm{i}}-\mathrm{t}_{\mathrm{i}-1}\right) * \mathrm{~A}\right] \\
& \text { donde, } \\
& \mathrm{P}_{\mathrm{i}-1}: \text { masa al tiempo i-1 }(\mathrm{kg}) \\
& \mathrm{P}_{\mathrm{i}}: \text { masa al tiempo i }(\mathrm{kg}) \\
& \mathrm{t}_{\mathrm{i}}: \text { tiempo i }(\mathrm{h}) \\
& \mathrm{t}_{\mathrm{i}-1}: \text { tiempo i-1 }(\mathrm{h}) \\
& \mathrm{A}: \text { área de la bandeja }\left(0,0484 \mathrm{~m}^{2}\right)
\end{aligned}
$$

\section{RESULTADOS Y DISCUSIÓN}

En las Figuras 2 y 3 se muestran las cinéticas de deshidratación obtenidas para las hojas de morera, las cuales siguen los patrones típicos de la deshidratación de los alimentos descritas por Geankoplis (1998). La pérdida de humedad al deshidratar materiales biológicos se describe generalmente en función del tiempo en términos de un decaimiento exponencial.

$\mathrm{Al}$ aumentar la temperatura del aire se incrementó la pendiente de la curva de deshidratación de la morera, implicando como consecuencia una disminución en el tiempo de secado (Figura 2). Esto ocurre por una combinación de dos factores. Primero, un aumento en la temperatura de secado provoca un incremento en la temperatura del producto y en el coeficiente de difusión del agua, consiguiendo que la velocidad de secado sea apreciablemente menor. A esto se suma que a mayor temperatura la humedad relativa del aire es menor, lo que aumenta su capacidad para absorber agua, favoreciendo la remoción de humedad (Bimbenet et al. 1985, Intermediate Technology Development Group 1998). 

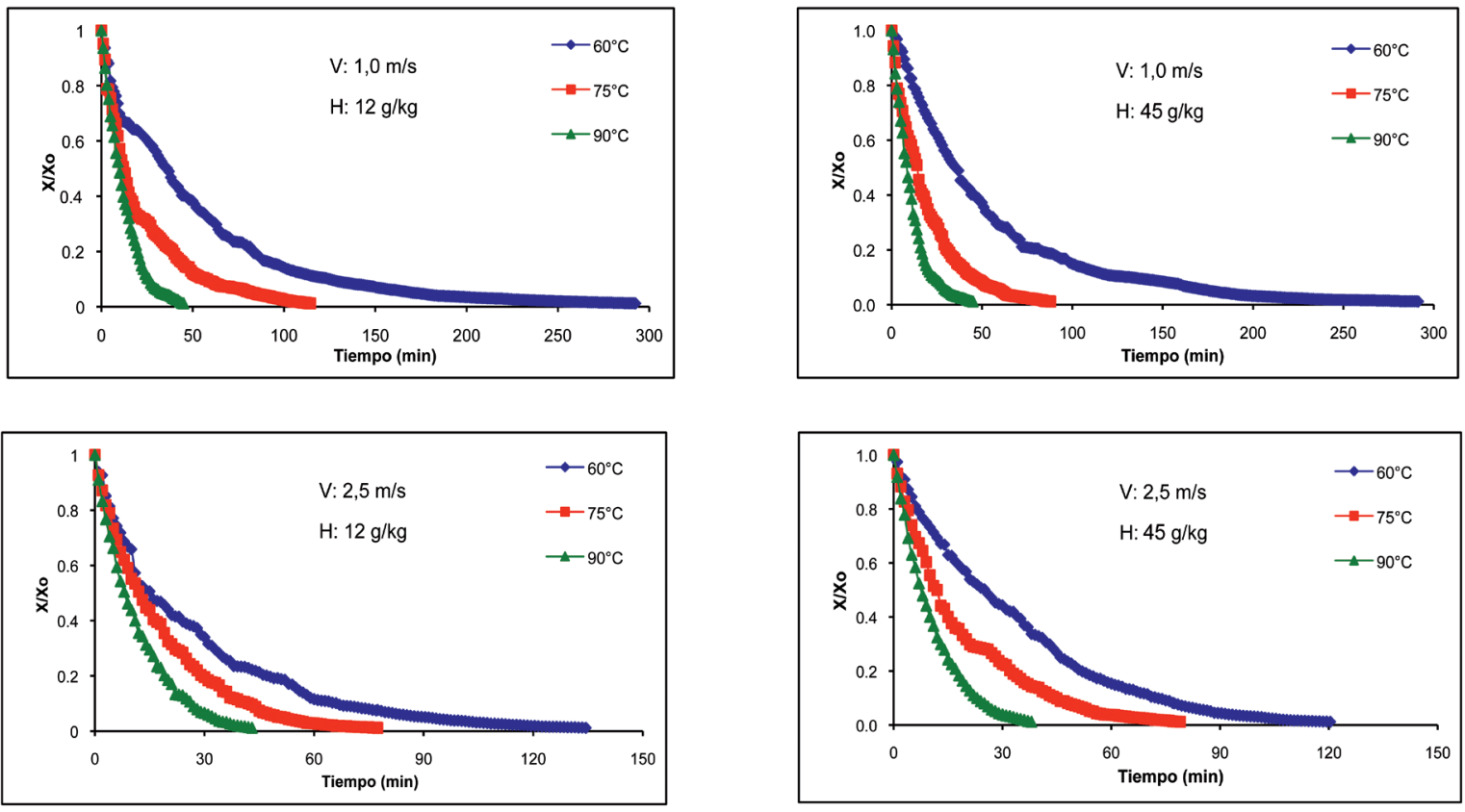

Figura 2. Efecto de la temperatura del aire sobre la cinética de deshidratación de las hojas de morera a dos velocidades y dos humedades del aire. V: velocidad, H: humedad en gramos de agua por kilogramo de aire seco. San José, Costa Rica. 2008.
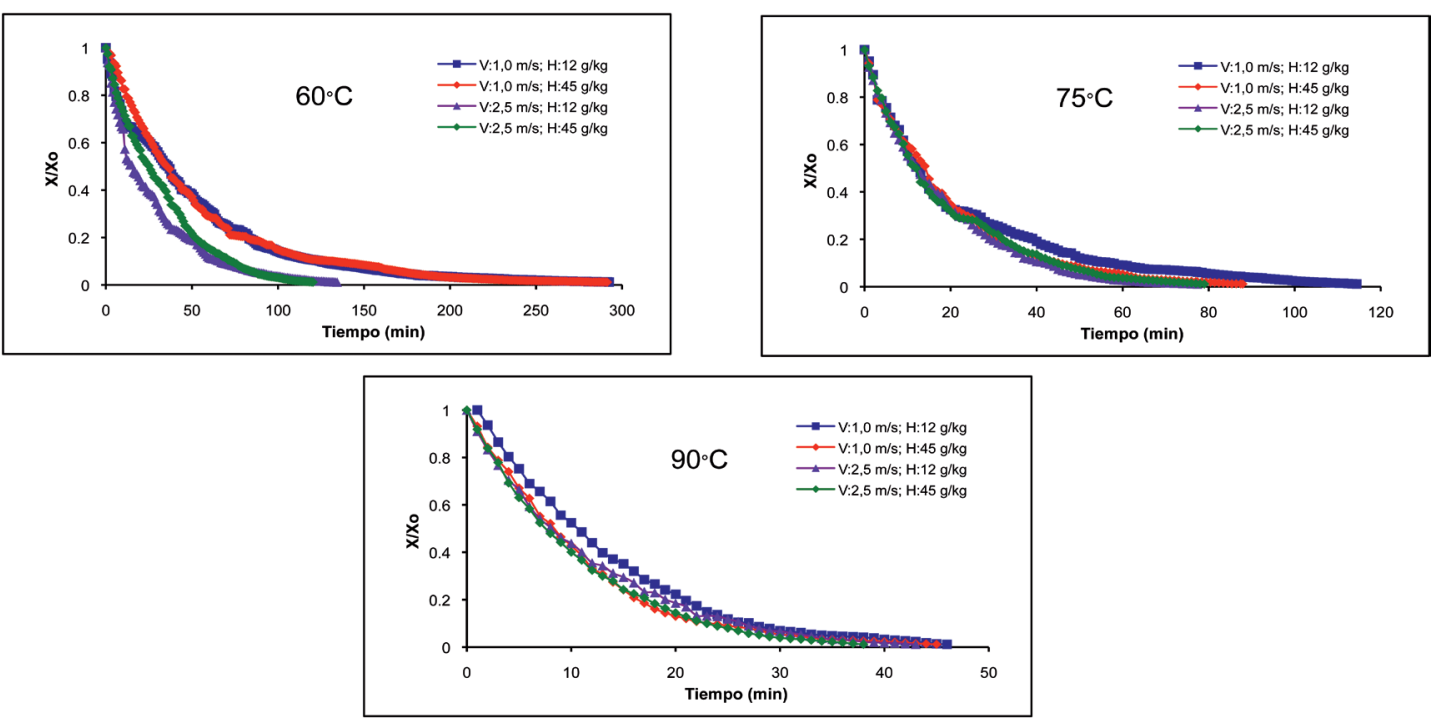

Figura 3. Efecto de la velocidad y la humedad del aire sobre la cinética de deshidratación de las hojas de morera a tres diferentes temperaturas de secado. V: velocidad, H: humedad en gramos de agua por kilogramo de aire seco. San José, Costa Rica. 2008. 
El aumento en la pendiente de la curva de secado fue más pronunciado cuando la temperatura se elevó de 60 ${ }^{\circ} \mathrm{C}$ a $75{ }^{\circ} \mathrm{C}$ que cuando pasó de $75{ }^{\circ} \mathrm{C}$ a $90{ }^{\circ} \mathrm{C}$.

La literatura enuncia diversos tipos de modelos matemáticos empleados en el análisis de curvas de deshidratación, donde para cada sustrato puede ajustar mejor un modelo que otro: Newton (o Lewis), Page, Page modificado, Henderson y Pabis, Henderson y Pabis modificado, logarítmico, dos términos, dos términos exponencial, Wang y Singh, Thompson, aproximación a la difusión, Verma, Midilli, y Chávez y Méndez (Waewsak et al. 2006, Akpinar y Bicer 2005, García et al. 2007). En este trabajo se evaluó el modelo de Newton que es el más comúnmente aplicado (Waewsak et al. 2006), y que corresponde con un decaimiento exponencial de la humedad en función del tiempo de secado. Este modelo ha sido empleado para modelar la deshidratación de vegetales como coco (Pineda 1993), culantro (Víquez 2001), palmito (Chacón 1998) y pejibaye (Ugalde 2002). Las predicciones obtenidas en el presente trabajo para el secado mostraron un excelente coeficiente de correlación (superior a 0,985 en todos los casos), lo que implica un proceso de deshidratación de las hojas de morera que se ajusta satisfactoriamente al modelo de Newton adoptado.

Un incremento de la velocidad del aire va unido con un incremento de la pendiente de la curva de deshidratación, y disminuyó en consecuencia la duración de la operación (Figura 3). Este comportamiento se fundamenta en el hecho de que al elevarse la velocidad del aire aumenta la eliminación de humedad superficial del producto, dado el mayor recambio de aire y la mayor remoción de agua que es arrastrada por este flujo (Singh y Heldman 1993).

El aumento de la humedad del aire tuvo un efecto similar a los incrementos en la temperatura y en la velocidad, pues disminuyó el tiempo de deshidratación de las hojas de morera (Figura 3). Según Geankoplis (1998), los materiales coloidales y fibrosos como vegetales y otros productos alimenticios, se contraen al eliminarse la humedad, lo que tiene como efecto el desarrollo de una capa superficial dura e impermeable al flujo de líquido o de vapor, lo que disminuyó la velocidad de secado. Uno de los factores que influye en la formación de esta capa es que, conforme el agua se mueve hacia la superficie, arrastra materiales solubles como azúcares y sales y, cuando el agua se evapora en la superficie, las sustancias solubles se acumulan en esta superficie deshidratada, lo cual impide el secado (Brennan 2006), debido al descenso de la difusividad del agua (McCabe 1991). Además, en muchos alimentos, si el secado se realiza a temperaturas muy altas, se forma en la superficie una capa de células contraídas que se adhieren unas a otras formando un sello (Panyawong y Devahastin 2007). Este fenómeno, denominado endurecimiento superficial, se constituye entonces en una barrera a la migración de la humedad (De Michelis et al. 2008). Para disminuir estos efectos del secado, es factible emplear aire húmedo, el cual, debido a su saturación, tiene la propiedad de humectar la cubierta impermeable de material seco que disminuye la transferencia de masa y de calor a través del producto (Geankoplis 1998), lo que puede explicar que la deshidratación de las hojas de morera se vea favorecida al disminuirse los efectos de la contracción y el endurecimiento. Adicionalmente, la forma aplanada de las hojas de morera hace que éstas se adhieran unas con otras, limitando el espacio para el paso del aire. Este proceso se pudo potenciar al emplear aire con baja humedad y contribuir a la menor tasa de deshidratación.

El análisis de los tiempos de secado permite comprender mejor el comportamiento de la deshidratación de las hojas de morera. Las variables de deshidratación evaluadas tuvieron un efecto significativo $(p<0,05)$ sobre el tiempo de deshidratación, lo mismo que fueron significativas todas las interacciones dobles y la interacción triple Temperatura*Velocidad* Humedad $(\mathrm{p}<0,05)$. Esto significa que el efecto de la temperatura sobre el tiempo de secado dependió de la velocidad y de la humedad del aire. Además, las réplicas no resultaron significativamente diferentes entre sí $(\mathrm{p}>0,05)$, implicando la existencia de poca variabilidad entre diferentes lotes de morera.

La interpretación de la interacción triple se puede inferir con base en la Figura 4. Se observa que para todas las velocidades y humedades el tiempo de deshidratación disminuyó con el aumento de la temperatura. Este efecto fue más pronunciado cuando se utilizó una velocidad del aire de $1 \mathrm{~m} / \mathrm{s}$, sobre todo entre los $60{ }^{\circ} \mathrm{C}$ y $75{ }^{\circ} \mathrm{C}$, sin embargo, la velocidad del aire de $2,5 \mathrm{~m} / \mathrm{s}$ generó tiempos de secado menores que los correspondientes a la velocidad de $1,0 \mathrm{~m} / \mathrm{s}$. Al aumentar la temperatura de deshidratación entre $60^{\circ} \mathrm{C}$ y $90^{\circ} \mathrm{C}$ se tuvo una reducción de un $65 \%$ en el tiempo de secado a la velocidad de 1,0 $\mathrm{m} / \mathrm{s}$ y una reducción de un $50 \%$ a la velocidad de 2,5 $\mathrm{m} / \mathrm{s}$. Un aire con $45 \mathrm{~g}$ agua $/ \mathrm{kg}$ aire seco (AS) produjo tiempos de secado levemente inferiores que el aire con una humedad de $12 \mathrm{~g} / \mathrm{kg}$ AS. Sin embargo, cuando se utilizó una velocidad de $2,5 \mathrm{~m} / \mathrm{s}$ no hubo diferencia 


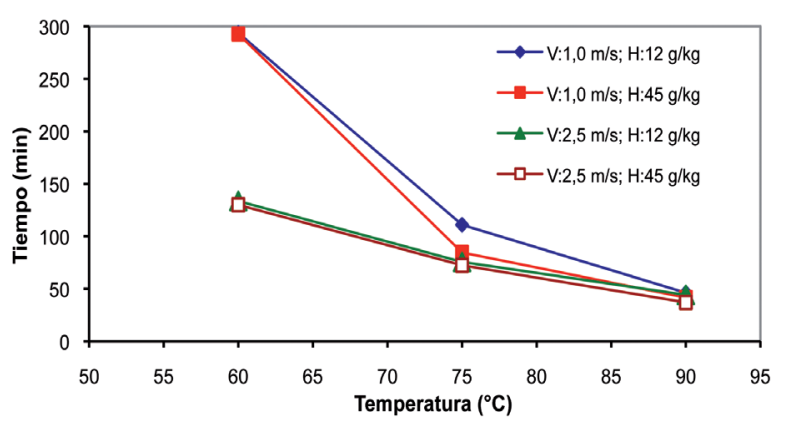

Figura 4. Efecto de la temperatura del aire sobre el tiempo de secado de las hojas de morera hasta un $5 \%$ de humedad, (H: humedad del aire en gramos de agua por kilogramo de aire seco, V: velocidad del aire). San José, Costa Rica. 2008.
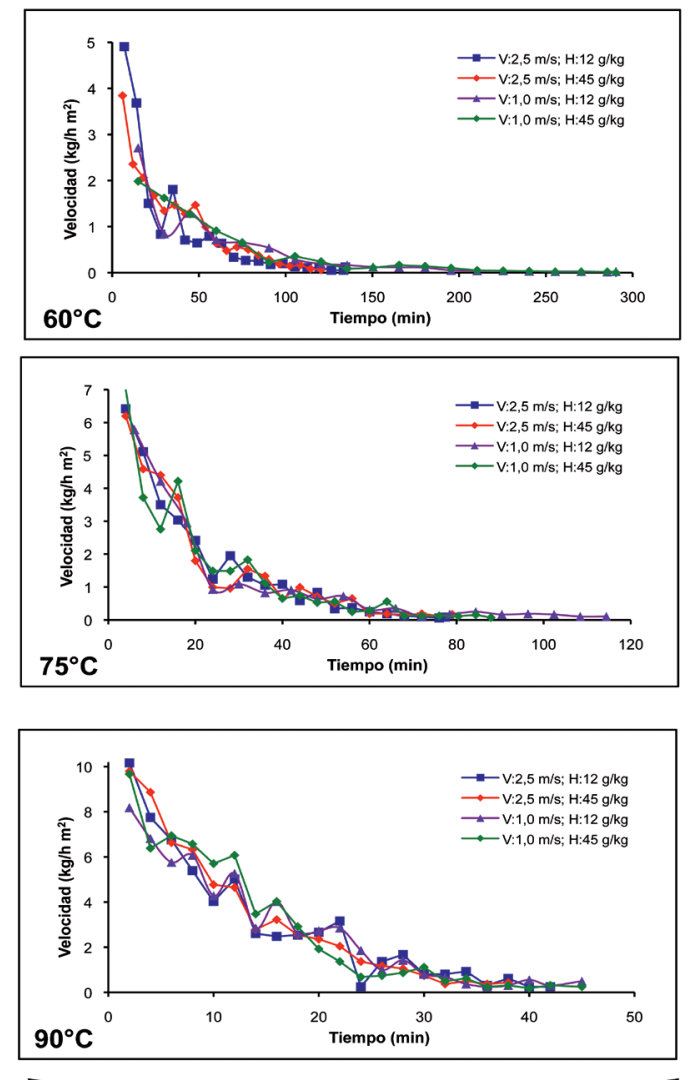

A. Velocidad vs. Tiempo en el tiempo de deshidratación obtenido con las dos humedades evaluadas. Finalmente, se observa que a 90 ${ }^{\circ} \mathrm{C}$ las variaciones en la velocidad o la humedad del aire no afectaron el tiempo de secado del forraje.

La Figura 5 muestra las curvas de velocidad de secado, que se caracterizan por una ausencia de los periodos de estabilización y de velocidad constante, y un comportamiento muy variable en la zona de velocidad decreciente que domina el proceso de deshidratación experimental, al igual que en el secado de otros materiales, como refiere Geankoplis (1998). Esta alta variabilidad implicó aumentos y disminuciones abruptas de la velocidad de secado, atribuibles a la escaza transferencia de calor y masa en algunos lapsos durante el secado, debido a la forma aplanada de las hojas de morera. A pesar de las fluctuaciones, las curvas de
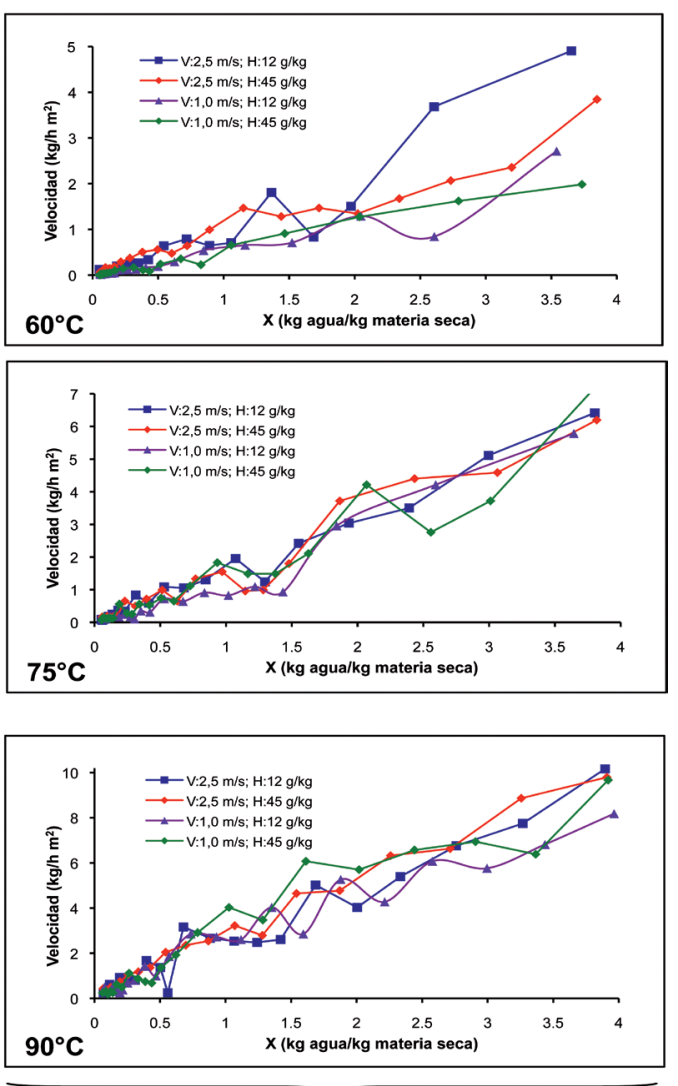

B. Velocidad vs. Humedad del producto

Figura 5. Gráficas de velocidad de secado de hojas de morera en función del tiempo y humedad del producto en base seca, para diferentes temperaturas, velocidades y humedades del aire. San José, Costa Rica. 2008. 
velocidad de secado en función del tiempo mostraron claramente un decaimiento exponencial, al igual que las curvas de humedad en función del tiempo de secado, y la relación de la velocidad en función de la humedad presenta una tendencia lineal, especialmente en las curvas obtenidas a $75^{\circ} \mathrm{C}$ y $90^{\circ} \mathrm{C}$.

Según la Figura 5, durante el proceso de secado se evidencian mayores tasas de velocidad de secado al aumentar la temperatura, en correspondencia con los menores tiempos del proceso que se generan con el aumento de la temperatura del aire y que fueron analizados anteriormente. Además, no se observa un efecto consistente de la velocidad y la humedad del aire sobre los gráficos de velocidad de secado de las hojas de morera.

Dado el gran efecto de las condiciones de secado sobre los tiempos de deshidratación de la morera, resulta de interés que estudios futuros evalúen el efecto de las condiciones de secado sobre las variaciones nutricionales y del color del forraje.

\section{AGRADECIMIENTOS}

Esta investigación contó con la colaboración de la Estación Experimental de Ganado Lechero Alfredo Volio Mata de la Universidad de Costa Rica, del Convenio de Apoyo Científico y Financiero con el organismo AIRE Développment, adscrito al IRD (Institut de Recherche pour le Développment) y del Centro Nacional de Ciencia y Tecnología de Alimentos (CITA) de la Universidad de Costa Rica.

\section{LITERATURA CITADA}

Akpinar, EK; Bicer, Y. 2005. Modelling of the drying of eggplants in thin-layers. International Journal of Food Science and Technology 40:273-281.

Alfaro, M; Martínez, R; Centeno, F; Araujo, G. 1997. Producción de leche y grasa láctea de vacas alimentadas con morera fresca (Morus alba) var. Kanva-2. Archivos Latinoamericanos de Producción Animal 5(1):139-143.

Bamikole, MA; Ikhatua, MI; Ikhatua, UJ; Ezenwa, IV. 2005. Nutritive value of mulberry (Morus spp.) leaves in the growing rabbits in Nigeria. Pakistan Journal of Nutrition 4(4):231-236.
Barbanti, D; Mastrocola D; Severini, C. 1994. Air drying of plums: a comparison among twelve cultivars. Sciences des Aliments 14(1):61-73

Belghit, A; Kouhila, M; Boutaleb, B.c. 2000. Experimental study of drying kinetics by forced convection of aromatic plants. Energy Conversion \& Management 41:1303-1321.

Bimbenet, JJ; Daudin, JD; Wolff, E. 1985. Air drying kinetics of biological particles. In: Proceeding Fourth International Drying Simposium, Kyoto. Washington, DC. 178-185.

Boschini, C. 2000. Calidad Nutricional de la Morera (Morus alba) cultivada y cosechada para uso en la alimentación de rumiantes (en línea). Consultado: 20 abril 2004. Disponible: http://www.fao.org/waisent/faoinfo/agricult/aga/agap/frg/mulberry/default.html

Boschini, C. 2003. Sustitución de alimento concentrado con morera fresca (Morus alba) aprovechables en vacas lecheras. Agronomía Mesoamericana 17(2):141-150.

Boschini, C. 2006. Nutrientes digeribles, energía neta y fracciones proteicas de la morera (Morus alba) en la dieta de vacas lecheras. Agronomía Mesoamericana 14(2):185-192.

Brennan, JG; Butters, JR; Cowell, ND; Lilley, AEV. 1998. Las operaciones de la ingeniería de los alimentos. 3 ed. Acribia. Zaragoza. 714 p.

Brennan, J.G. 2006. Food processing handbook. WileyVCH, Weinheim, Germany. 607 p.

Chacón, A. 2004. Perspectivas actuales de la proteína unicelular (SCP) en la agricultura y la industria. Agronomía Mesoamericana 15(1):93-106.

Chacón, I. 1998. Estudio del efecto de la temperatura, humedad y velocidad del aire en el secado con aire caliente del palmito de pejibaye (Bactris gasipaes) y de su molienda para la obtención de un producto deshidratado en polvo. Tesis Lic. Tecnología de Alimentos. Escuela de Tecnología de Alimentos, Universidad de Costa Rica. San José, Costa Rica. 69 p.

De Michelis, A; Pirone, BN; Vullioud, MB; Ochoa, MR; Kesseler, AG; Márquez, CA. 2008. Cambios de volumen, área superficial y factor de forma de Heywood durante la deshidratación de cerezas (Prunus avium). Ciência e Tecnología de Alimentos 28(2):317-321.

Doymaz, I. 2004. Drying kinetics of white mulberry. Journal of Food Engineering 16(3):341-346.

Duke, J. 1983. Morus alba L. Handbook of energy crops. Purdue University (online). Consultado: 25 set. 2004. 
Disponible en: Www.hort.purdue.edu/newcrop/duke_ energy/Morus_alba.htlm.

Estévez, OV; Pedraza, RM; Guevara, RV; Parra, CE. 2004. Composición química y degradabilidad ruminal del follaje de tres variedades de Polyscias guilfoylei en la época seca. Pastos y Forrajes 27(2):177-181.

García, DE; Ojeda, F. 2004. Evaluación de los principales factores que influyen en la composición fotoquímica de Morus alba (Linn.): II Polifenoles totales. Pastos y forrajes 27(1):59-64.

García, SV; Schmalko, ME; Tanzariello, A. 2007. Isotermas de adsorción y cinética de secado de ciertas hortalizas y aromáticas cultivadas en Misiones. RIA 36(1):115-129.

Geankoplis, C. 1998. Procesos de transporte y operaciones unitarias. 3 ed. Cecsa, México, D.F. 1008 p.

Intermediate Technology Development Group. 1998. Libro de consulta sobre tecnologías aplicadas al ciclo alimentario: técnicas de secado. ITDG, Perú. 72 p.

Jirakitkul, N. 2007. Microwave vacuum drying of mulberry leaves (online). School of Environment, Resouces and Development (SERD), Asian Institute of Technology. Pathumthani, Thailand. Consultado: 9 junio 2008. Disponible: Http://www.library.ait.ac.th/ThesisSearch/ showDetails.asp?id=14295

Krokida, MK; Karanthanos, VT; Maroulis, ZB; Marinoskouris, D. 2003. Drying kinetics of some vegetables. Journal of Food Engineering 59(4):391-403.

McCabe, WL; Smith, JC; Harriott, P. 1991. Operaciones unitarias en ingeniería química. 4 ed. McGraw-Hill, Madrid. 1176 p.

Murakami, S. 2003. Mulberry leaves powder manufacturing method (US Patent 6536689). Freepatentsonline (en línea). Consultado: 9 junio 2008. Disponible: http:// www.freepatentsonline.com/6536689.html

Nieves, D; Aranque, H; Teran, O; Silva, L; Gonzalez, C; Uzcategui, W. 2006. Digestibilidad de nutrientes del follaje de morera (Morus alba) en conejos de engorde. Revista Científica 16(4):364-370.

Ortiz, G. 1992. Efecto de la alimentación con pasto King Grass (Pennisetum purpureum) suplementado con diferentes niveles de follaje de morera (Morus alba) y de banano verde (Musa sp.) sobre la producción de leche de cabra. Tesis Lic. Ing. Zootecnista. Escuela de Zootecnia, Universidad de Costa Rica. San José. 45 p.

Panyawong, S; Devahastin, S. 2007. Determination of deformation of a food product undergoing different drying methods and conditions via evolution of a shape factor. Journal of Food Engineering 78(1):151161.

Piccioni, M. 1970. Diccionario de alimentación animal. Acribia, Zaragoza. p. 492-494.

Pineda, ML. 1993. Desarrollo de un producto deshidratado a partir de coco (Cocos nucifera Linn.). Tesis Lic. Tecnología de Alimentos. Carrera Interdisciplinaria en Tecnología de Alimentos, Universidad de Costa Rica. San José. 162 p.

Rodríguez, C; Arias, R; Quiñones, J. 1994. Efecto de la frecuencia de poda y el nivel de fertilización nitrogenada, sobre el rendimiento y calidad de la biomasa de Morera (Morus alba) en el trópico seco de Guatemala. Árboles y Arbustos Forrajeros de Centroamérica. Serie Técnica. CATIE. Informe Técnico No 236. Volumen II. p. 515-528.

Sánchez, M. 1999. Morera: un forraje excepcional disponible mundialmente. FAO, Roma. (en línea) Consultado: 23 nov. 2004. Disponible en: http://www.cipav.org.co/ redagrofor/memorias99/SanchezM.htm

Sharma, SK; Mulvaney, SJ; Rizvi, SSH. 2003. Ingeniería de alimentos: operaciones unitarias y prácticas de laboratorio. Limusa Wiley. México. 348 p.

Singh, RP; Heldman, DR. 1993. Introducción a la ingeniería de los alimentos. Acribia, Zaragoza. 564 p.

Ugalde, H. 2002. Estudio de la deshidratación del pejibaye (Bactris gasipaes H.B.K) para la elaboración de harina y su utilización en la fórmula de una premezcla para queques. Tesis Lic. Tecnología de Alimentos. Escuela de Tecnología de Alimentos, Universidad de Costa Rica. San José, Costa Rica. 100 p.

Vega, A; Tello, C; Lemus, R. 2007. Simulación matemática del proceso de secado de la gracilaria chilena (Gracilaria chilensis). Ingeniare: Revista Chilena de Ingeniería 15(1):55-64.

Víquez, JF. 2001. Determinación de las condiciones adecuadas para el secado de culantro (Coriandrum sativum L.) con aire caliente. Tesis Lic. Tecnología de Alimentos. Escuela de Tecnología de Alimentos, Universidad de Costa Rica. San José. 94 p.

Waewsak, J; Chindaruksa, S; Punlek, C. 2006. A mathematical modeling study of hot air drying for some agricultural products. Thammasat International Journal of Science and Technology 11(1):14-20. 
